Journal of Computer Science 7 (1): 12-16, 2011

ISSN 1549-3636

(C) 2011 Science Publications

\title{
Pattern Based Communication System and Its Performance Characteristics Compared to the Matched Filtering
}

\author{
Ozgur Orcay and Berk Ustundag \\ Department of Computer Engineering, \\ Faculty of Computer Engineering, \\ Istanbul Technical University, 34469, Maslak, Istanbul, Turkey
}

\begin{abstract}
Problem Statement: Pattern Based Communication System (PBCS) aims to handle two prominent restrictions in communication lines. These are the inefficient utilization of the bandwidth under the spectrum limitations and sensitivity of the data transmission rate to the variation of Signal to Noise Ratio (SNR). Approach: The key point of PBCS is to construct the optimal communication signals, which consist of patterned data that can be recovered by the cognitive receiver, for a successful data transmission even in low SNR and high data bit rate. Result: In this study, performance of the PBCS is evaluated by the comparison with Matched Filtering (MF). Conclusion: This comparison shows that transmission performance of the PBCS is higher than MF due to its dynamic and flexible operation capability under variation of spectral conditions.
\end{abstract}

Key words: Artificial neural network, cognitive radio, noise immunity, pattern recognition, spectrum management

\section{INTRODUCTION}

There are two crucial aspects those define fundamental restrictions in data transmission channels. One of them is the spectral bandwidth limitation and the other is robustness to noise at error free data transmission speeds. Pattern Based Communication System (PBCS) (Ustundag and Orcay, 2008) targets to handle these restrictions. It involves a new modulation and encoding technique that adaptively uses collection of weakly correlated signal pattern sets called glossaries. Each glossary in the glossary space is indexed regarding to classification of two components: The transmission capability under the specified noise suppression in terms of Signal to Noise Ratio (SNR) and Link Spectral Efficiency (LSE) (bps/Hz), which consists of the utilized spectral bandwidth $(\mathrm{Hz})$ by the test signal pattern and its data bit rate (bps).

As proposed by the Shannon Limit Theorem (Eq. 1) (Shannon, 1948), the channel capacity is directly proportional to the spectral bandwidth and SNR. In order to maximize the channel capacity both parameters should be optimized:

$\mathrm{C}=\mathrm{W} \times \log _{2}\left(1+\frac{\mathrm{S}}{\mathrm{N}}\right)$
Where:

$\mathrm{C}=$ An information rate in bits per second

$\mathrm{W}=$ The spectral bandwidth of the channel in hertz

$\mathrm{S}=$ The total signal power

$\mathrm{N}=$ The total noise power in the bandwidth

In PBCS, the transmitter uses the free SNR capacity and the free spectral bandwidth of communication channel, dividing the data bit rate to the utilized spectral bandwidth to come up with the LSE. These two values correspond to a specific glossary in the glossary space. In the receiver, the disarrangements caused by possible delay and reflections in the communication medium are corrected via a synchronization process that puts the patterns in correct order. Then these patterns are fed into the recognition layer, namely Artificial Neural Network (ANN). Having been trained for all possible sets of glossaries, the ANN decomposes the incoming noise added signal pattern into the associated binary code. The information is reconstructed by concatenating these binary codes. Matched Filtering (MF) (Turin, 1960) has some similarities with PBCS due to its nature in the physical layer. In this paper, performance of PBCS is evaluated by the comparison with MF.

Remaining sections of the paper are organized as follows: Basics of the PBCS are summarized in section

Corresponding Author: Ozgur Orcay, Department of Computer Engineering, Faculty of Computer Engineering,

Istanbul Technical University, 34469, Maslak, Istanbul, Turkey 
PBCS Model. In section Materials and Methods, transmitter/receiver structures of the PBCS are introduced for comparison with commonly known $\mathrm{MF}$ structure. Simulation results of this comparison are presented in section Simulation Results and the conclusion is drawn in the last section.

PBCS model: A prominent subject under the Cognitive Radio (CR) (Mitola, 1999) concept is spectrum sensing, for which extensive work has been performed in the literature. This work includes Matched Filtering (Turin, 1960), Waveform-based sensing, Cyclostationary-based Sensing, Energy Detector-based Sensing and Radio Identification (Arslan, 2007). Among these, MF is the closest method to PBCS. The most significant disadvantage in the practical use of MF is the fact that the information about bandwidth, operating frequency, modulation type and order, pulse shaping, frame format etc. should also be known by the receiver, whereas proposed system overcomes this disadvantage, since the information is embedded into the recognition process of ANN. On the other hand, there are various methods that utilize ANN to make spectrum sensing in CR. Palicot and Roland (2003) have managed to successfully determine the bandwidth and its shape by using Radial-based Neural Network. In another study, researchers (Gandetto et al., 2004) have used FeedForward Back Propagation Neural Networks and Support Vector Machine with Radial Bases Function to make time-frequency analysis. Finally, Fehske et al. (2005) have used Spectral Correlation Density and function to perform Multi-layer Perceptron (MLP) to sense the spectrum. As opposed to these methods, PBCS develops its own modulation technique by using Amplitude Frequency Phase Shift Keying (AFPSK) and the data communication is achieved when the signals constructed through this method are recognized by ANN. Briefly, the novelty point of PBCS is using ANN in physical layer to recognize its own communication signal patterns in pre-defined glossaries. The fundamental reason for selecting ANN as the recognition layer of the system is the sensitivity of ANN while recognizing the amplitude, frequency and phase changes in the communication signal.

Moreover, from the CR perspective, the PBCS has the objective of optimizing the SNR Vs. LSE relationship of the communication system by making dynamic band utilization through pre-defined glossaries, which have distinct characteristics. In fact, this relationship can be seen clearly in the following equation of Energy per bit to Noise Power Spectral Density Ratio $\left(\mathrm{E}_{\mathrm{b}} / \mathrm{N}_{0}\right)$ :
$\frac{\mathrm{E}_{\mathrm{b}}}{\mathrm{N}_{0}}=\frac{\mathrm{S} / \mathrm{C}}{\mathrm{N} / \mathrm{W}}=\frac{\mathrm{S} / \mathrm{N}}{\mathrm{C} / \mathrm{W}}=\frac{10^{\mathrm{SNR} / 10}}{\mathrm{LSE}}$

The main purpose of this study can be expressed as:

to minimize $\int_{\left(\mathrm{E}_{\mathrm{b}} / \mathrm{N}_{\mathrm{o}}\right)_{\min }}^{\left(\mathrm{E}_{\mathrm{b}} / \mathrm{N}_{\mathrm{o}}\right)_{\max }} \mathrm{f}_{\mathrm{BER}}(\mathrm{x}) \mathrm{dx}$
with subject to $\left(\mathrm{W}_{\mathrm{g}} £ \mathrm{~W}_{\text {free }}\right) \grave{\mathrm{U}}\left(\mathrm{C}_{\mathrm{g}}{ }^{3} \mathrm{C}_{\text {req }}\right) \grave{U}\left(\mathrm{SNR}_{\mathrm{g}} £ \mathrm{SNR}_{\text {free }}\right)$

where function $\mathrm{f}_{\mathrm{BER}}$ defines the function of BER upon $\mathrm{E}_{\mathrm{b}} / \mathrm{N}_{0}$ (Fig. 3). One of the constraints $\mathrm{W}_{\mathrm{g}}$ that represents the spectral bandwidth utilization of the active glossary. It should be lower than the free spectral capacity of the utilized communication channel $\left(\mathrm{W}_{\text {free }}\right)$. Another constraint that is the data bit rate capacity of active glossary called as $C_{g}$ that should be equal to or higher than the user request $\left(\mathrm{C}_{\text {req }}\right)$. At the end, $\mathrm{SNR}_{\mathrm{g}}$ points to the robustness of the glossary against to noise in terms of $\mathrm{dB}$. The selected glossary should be robust enough to the channel noise level $\left(\mathrm{SNR}_{\text {free }}\right)$. Under these constraints, PBCS aims to minimize the total area under the graph (Fig. 3), which is drawn for BER Vs. $\mathrm{E}_{\mathrm{b}} / \mathrm{N}_{0}$ space. Many different glossaries can be constructed in PBCS by the usage of AFPSK. The mathematical expression of the constructed signal pattern is:

$S_{i, j}(t)=f\left(A_{i, j}(t), f_{i, j}(t), \Phi_{i, j}(t), t\right)$ for $i=1 \ldots k ; j=1 \ldots 1$

where, $S_{i, j}$ represents $j^{\text {th }}$ signal pattern of the $i^{\text {th }}$ glossary. $\mathrm{k}$ and 1 show the number of the glossary and the level of the active glossary in PBCS, respectively (i.e. $n=\log _{2} 1$ for $n$-bit glossary in Fig. 1). Moreover, A, f and $\Phi$ points the shift keying borders of amplitude, frequency and phase in an order.

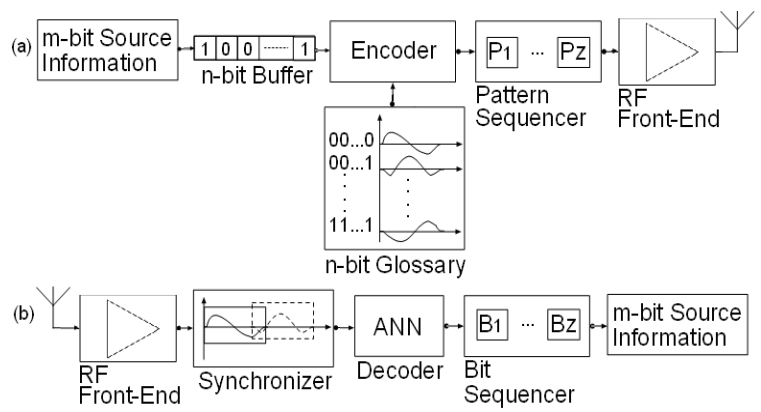

Fig. 1: PBCS Structure: (a) Transmitter, (b) Receiver 
PBCS utilizes the user-defined $\mathrm{R}$ under limited spectral bandwidth $(\approx$ LSE) and the free SNR capacity in communication channel as an input. Being a function of these two values as shown in equation 2, $\mathrm{E}_{\mathrm{b}} / \mathrm{N}_{0}$ ratio is used to determine the appropriate glossary to be transmitted from the glossary space. Since the glossary space is known by both, the transmitter and the receiver. The ANN decodes the information and offers it to user service. At this stage, the most important problem is the lack of synchronization between the patterns. This problem can be solved by sending additional synchronization patterns that is a commonly used method in literature. Moreover, since the sampling frequency of received patterns is proportional to the baseband and the assigned bandwidth, Direct RF sampling is preferably applied together with band-shifting at the RF Front-end as explained by Akos et al. (1999). Otherwise baseband frequency dramatically increases sampling frequency and this reduces feasibility of the method.

\section{MATERIALS AND METHODS}

As in all communication systems, the digital information is modulated in the transmitter and demodulated in the receiver based on a method. The modulation method in PBCS depends on the signal pattern construction. On the other hand, the demodulation corresponds to the offline pre-training of the ANN.

Transmitter structure: The transmitter structure of PBCS is shown in Fig. 1a. The sequence of the binary bits, which constitutes the main information, is fed into a buffer, according to the size of the glossary. At this stage, the n-bit sequence is matched with the n-bit glossary in order to be given to the pattern sequencer, which concatenates these patterns in sequence. Then the constructed pattern sequence is transmitted through RF front-end. As seen, by not requiring any modulation and/or coding layer and by affecting directly the performance of the system, glossary construction forms the basis of PBCS.

It is assumed that in order to construct the glossary space, three fundamental features of the communication signal need to be shifted. All possible outcomes in the Sinusoidal Pattern Envelope Construction (SPEC) space are formed by the changes in them. The envelope combinations for \pm 2 levels and 6 depth layers are given as an example. One of the alternatives is given in bold in Fig. 2. Three possible alterations between each depth layer can be positive or negative quarter sinus or constant level. Since the changes in the signal features form the basis of the pattern system, they should be as uncorrelated as possible. The vertical levels in the SPEC space represent the maximum and the minimum values that any of the signal characteristics can take. For instance, in a case of frequency change is chosen to be between 2410 and $2414 \mathrm{MHz}$, they are assigned to $-\mathrm{L} 2$ and $+\mathrm{L} 2$ respectively. As a fundamental necessity of the RF communication, the signals should end at its starting point in order to have a zero average power density. The process of the signal pattern construction aims to handle the defined constraints.

Receiver structure: In the receiver of PBCS, the signal distorted in the communication medium arrives to the RF front-end, where, after a synchronization process, it will be fed into the ANN as an input. Since ANN is pre-trained for the patterns, it can recover the distorted signal into known patterns, and outputs the associated bit sequences. These bit sequences are concatenated by the Bit Sequencer layer in order to come up with the original information. This procedure is described in Fig. 1b. ANN design needs the training and the test sets. In the training phase, the ANN is trained offline according to the glossary patterns, which are constructed by SPEC algorithm. These signal patterns are distorted in the communication medium and they are called to the test set. ANN that was first proposed by Haykin (1999) with the name of MLP achieved successful results in complex classification and pattern recognition problems. Therefore, ANN is used in order to recognize the signal patterns, which are constructed based on the RF signal construction principles in this study.



Fig. 2: Sinusoidal pattern envelope construction space 


\section{RESULTS}

MATLAB is used as a simulation tool for extracting the $\mathrm{E}_{\mathrm{b}} / \mathrm{N}_{0}$ Vs. BER graphs (Fig. 3) of PBCS and MF. The constructed signal patterns are taught to the single hidden layered MLP. The number of neurons at hidden layer is determined by the cross-validation. The number of neuron in input and output layers equal to the number of sample per pattern and the amount of pattern in glossary respectively. The learning rate is adjusted in an adaptive manner (additive increment/multiplicative decrement) and the momentum coefficient is used as 0.5 . The activation function is selected as the sigmoid with constant "a" is 0.6 (Eq. 5):

$\phi(v)=1 /(1+\exp (-\mathrm{a} \times v))$

The recognition rate of ANN can be calculated by "Recognition Rate $(\%)=100 \times(1-(\mathrm{n} \times \mathrm{BER}))$ ", where, n represents the bit level of the active glossary. The set of patterns inside the glossaries are used as the training set. Additive White Gaussian Noise combined patterns are used as the members of the test set. In this study, all possible transitions between two signal patterns in the glossary are taken into account in the test pattern sequence. Thus, the calculation of the spectral bandwidth utilization is performed most realistically.

The performance of PBCS with unique glossaries and of MF combined with BPSK is compared in the BER Vs. $\mathrm{E}_{\mathrm{b}} / \mathrm{N}_{0}$ space. Regarding to equation 3 , the total area under each of the above graphs indicates the overall system performance under different noise and data bit rate levels. Overall performance of the 1-bit encoded PBCS is at least $70 \%$ higher than MF combined with BPSK.

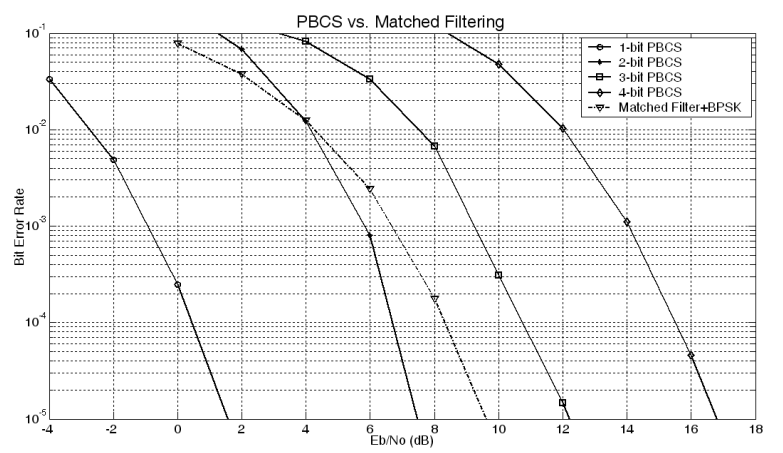

Fig. 3: The performance comparison between PBCS and Matched Filtering
This performance is calculated for the $\mathrm{E}_{\mathrm{b}} / \mathrm{N}_{0}$ range of $4 \mathrm{~dB}$ to $+10 \mathrm{~dB}$ and with respect to $10^{-5}$ BER offset value. On the other hand, 2-bit encoded PBCS demonstrates approximately $10 \%$ higher overall performance than the $M F$ in the $E_{b} / N_{0}$ range of $0 \mathrm{~dB}$ to $+10 \mathrm{~dB}$. This is also meaningful that BPSK combined MF can be considered as a sort of 2-bit encoded system as its PBCS equivalent. Overall performances for 3-bit and 4-bit encoded PBCS are worse than MF.

\section{DISCUSSION AND CONCLUSION}

The objective of Pattern Based Communication System (PBCS) is to improve data transmission performance under bandwidth limitations. Constructed signal patterns are matched with the binary information by the PBCS transmitter. The noisy signal is recovered by the Artificial Neural Network at the receiver side. The output is then concatenated by the Bit Sequencer to obtain the original information. This study draws a comparison between PBCS and Matched Filtering (MF) that is the most associated method in the literature. A performance measure function that refers to minimization of the area under the BER Vs. $\mathrm{E}_{\mathrm{b}} / \mathrm{N}_{0}$ graph is described for this purpose. Due to PBCS's nature, its performance can be improved by switching between the glossaries regarding to observed spectral conditions in an adaptive manner. This part of study is an ongoing work.

\section{REFERENCES}

Akos, D. M., M. Stockmaster, J. B. Y. Tsui, and J. Caschera, 1999. Direct bandpass sampling of multiple distinct RF signals. IEEE Trans. Commun., 47: 983-988. DOI: 10.1109/26.774848

Arslan, H., 2007. Cognitive Radio, Software Defined Radio, and Adaptive Wireless Systems. Springer, ISBN: 978-1-4020-5541-6

Fehske, A., J. Gaeddert and J. Reed, 2005. A new approach to signal classification using spectral correlation and neural networks. Proceeding of the IEEE International Symposium on New Frontiers in Dynamic Spectrum Access Networks, Baltimore, Maryland, pp: 144-150, DOI: 10.1109/DYSPAN.2005.1542629

Gandetto, M., M. Guainazzo, and C. S. Regazzoni, 2004. Use of time-frequency analysis and neural networks for mode identification in a wireless software-defined radio approach. EURASIP J. Applied Signal Process., 2004: 1778-1790. DOI: $10.1155 / \mathrm{S} 1110865704407057$ 
Haykin, S., 1999. Neural Networks: A Comprehensive Foundation. Upper Saddle River, Prentice Hall, NJ., ISBN: 0132-73350-1

Mitola, J., 1999. Cognitive Radio for Flexible Mobile Multimedia Communications. IEEE Mobile Multimedia Commun., 3-10. DOI: 10.1109/MOMUC.1999.819467

Palicot, J. and C. Roland, 2003. A new concept for wireless reconfigurable receivers. IEEE Commun. Maga., 41: 124-132. DOI: 10.1109/MCOM.2003.1215649
Shannon, C.E., 1948. A mathematical theory of communication. Bell Syst. Techn. J., 27: 279-423.

Turin, G.L., 1960. An introduction to matched filters. IRE Trans. Inform. Theory, 311-329. DOI; 10.1109/TIT.1960.1057571

Ustundag, B. and O. Orcay, 2008. Pattern based encoding for cognitive communication. Proceeding of the IEEE International Conference on Cognitive Radio Oriented Wireless Networks and Communications, Singapore, pp: 1-6. DOI: 10.1109/CROWNCOM.2008.4562494 